\title{
Variability of reciprocal aiming movements during standing: The effect of amplitude and frequency
}

\author{
Frédéric Danion $^{\mathrm{a}, *}$, Marcos Duarte $^{\mathrm{b}}$, Marc Grosjean ${ }^{\mathrm{c}}$ \\ ${ }^{a}$ UMR 6559 Mouvement et Perception, Université de la Méditerranée, CNRS, Faculté des Sciences du Sport, \\ 163 avenue de Luminy, 13288 Marseille Cedex 09, France \\ ${ }^{\mathrm{b}}$ Universidade de São Paulo, Escola de Educação Física e Esporte, São Paulo, Brasil \\ ${ }^{\mathrm{c}}$ Max Planck Institute for Human Cognitive and Brain Sciences, Department of Psychology, Munich, Germany
}

Received 2 July 2004; received in revised form 28 December 2004; accepted 20 January 2005

\begin{abstract}
This study investigated the variability of the center of pressure (COP) trajectory during voluntary whole-body oscillations. While standing upright on a force platform, eight subjects leaned forward and backward so as to perform reciprocal aiming movements with their COP at a prescribed frequency $(F)$ and amplitude $(A)$ using online visual feedback of their COP location. A total of $25 F-A$ combinations were tested for each subject $(3<A<9 \mathrm{~cm}$, and $0.35<F<1.35 \mathrm{~Hz})$. Spatial and temporal variability of the COP was assessed by computing the standard deviation (SD) and coefficient of variation $(\mathrm{CV})$ of the amplitude (trough to peak) and frequency (peak to peak) of the COP cycles within each trial, respectively. The results revealed that all variability indices depended on the prescribed $F$ and $A$. Concerning the effect of spatial constraints on spatial variability, SD spatial increased as a function of $A$, while CV spatial decreased as function of $A$. A similar pattern was observed with respect to the effect of temporal constraints on temporal variability (SD temporal increased as a function of $F$, while CV temporal decreased). As for "cross-over" effects, there was an effect of $F$ on spatial variability, such that SD spatial and CV spatial were minimal at $0.6 \mathrm{~Hz}$. For the "cross over" effect of $A$ on temporal variability, both SD spatial and CV spatial decreased as a function of $A$. Across the experimental conditions, there were weak or no correlations between variability in the time and space domain. Comparisons with an earlier study on human gait (Danion F, Varraine E, Bonnard M, Pailhous J. Stride variability in human gait: the effect of stride frequency and stride length. Gait Posture 2003;18:69-77) suggest that the effects of spatial constraints are relatively task independent, whereas the effects of temporal constraints depend on the nature of the motor task that is performed.
\end{abstract}

(C) 2005 Elsevier B.V. All rights reserved.

Keywords: Human movement; Posture; Center of pressure; Variability; Amplitude; Frequency

\section{Introduction}

In laboratory tasks and in daily activities, humans sometimes need to repeat movements in a sequence. Despite common features across successive movements, humans can never repeat exactly the same movement, be it in spatial or temporal terms. This intrinsic variability of human movement has always been a critical issue in motor control and all theoretical accounts of motor behavior either implicitly or explicitly consider variability (for a review, see [20]).

\footnotetext{
* Corresponding author. Tel.: +33 04911722 78; fax: +33 0491172252 . E-mail address: danion@laps.univ-mrs.fr (F. Danion).
}

Although a large number of studies have already investigated variability in movement, very few of them have considered the following issues. First, despite the richness of the human motor repertoire, the study of variability in voluntary movement has mainly dealt with rapid singleaiming movements, such as the production of spatially oriented movements with the arm $[22,17,6]$ or the production of isometric force pulses $[18,19]$. Less attention has been paid to characterizing variability in voluntary cyclical movements, especially in the context of whole-body movements. Second, although movements are performed simultaneously in the time and space domain, most research has focused on one dimension at a time. For example, it is unclear whether spatial and temporal variability change in concert with respect 
to various movement parameters. Third, very few studies have manipulated simultaneously spatial and temporal constraints. Therefore, the possibility that temporal constraints affect spatial variability or that spatial constraints affect temporal variability has been poorly investigated.

In a recent study, Danion et al. [5] investigated the variability of a cyclical whole-body movement: human gait. Specifically, they assessed the spatial and temporal variability of the stride with respect to changes in stride length and stride frequency. The results of that study showed that: (1) spatial and temporal variability tended to increase in concert with respect to stride parameters; (2) stride variability was minimal at $1 \mathrm{~Hz}$; and (3) stride variability decreased with larger strides. However, because the human motor repertoire is rather large, it is unclear whether these results (i.e., obtained for gait) would generalize to other whole-body movements. The main goal of the present study was to examine whether a similar pattern of results would also be obtained in a postural task. The use of a postural task seemed adequate for the following reasons. First, during upright posture, body sway is a typical example of oscillatory whole-body movements. Second, the ability to maintain balance during upright posture is a vital function for humans [27]. Third, the measurement of postural aiming movements using visual feedback of the center of pressure (COP) location, as will be examined in the present study (see below for details), is a common tool in the rehabilitation of patients with impaired balance $[24,10,11,21,1,7]$ as well as in theoretical studies $[8,14]$. Finally, studies of postural sway have formed the experimental basis for several theories of postural stabilization $[3,27,28,13,12,15]$.

The impetus for exploring variability in a postural task was based on an earlier study by Danion et al. [4] in which subjects performed reciprocal-pointing movements during upright posture. In that study, they determined how fast and accurate people could displace their COP location using online visual feedback. The results showed that for a given COP movement amplitude, movement time increased in a linear fashion with increases in the index of difficulty of the movement (i.e., with decreases in target size, see [9]). There was also the presence of a scaling effect on movement time in that the slope of the linear functions tended to increase with decreases in movement amplitude. In other words, it seemed that subjects had more difficulty in controlling small as opposed to large COP movements.

In the present study, a task in which COP movements were spatially and temporally constrained was employed. Subjects were asked to perform COP movements at a prescribed frequency and amplitude. With respect to the effect of amplitude and frequency on COP variability, the following hypotheses were formulated. First, if small COP movements are more difficult to control (cf. [4]), (relative) spatial variability should decrease as a function of movement amplitude. Second, if spatial and temporal variability co-vary in the same way as in human gait [5], temporal variability should also decrease as a function of movement amplitude. Third, Danion and colleagues previously showed that stride variability was minimal at $1 \mathrm{~Hz}[5]$ and proposed that this frequency was optimal because it corresponds to the "resonance frequency" at which the amount of energy needed to sustain the oscillation of the lower limbs is minimal. It was hypothesized that if a resonance frequency exists for the whole body during postural oscillations, movement variability should be minimal at this frequency. Given the larger inertia of the whole body (as compared to the lower limbs), this optimal frequency was expected to be below $1 \mathrm{~Hz}$ in the present task.

\section{Method}

\subsection{Subjects}

Eight subjects participated in this experiment, 5 men and 3 women. The mean subject age, height, and mass, were $28.5 \pm 6.7$ year, $174 \pm 10 \mathrm{~cm}, 66.8 \pm 14.5 \mathrm{~kg}$, respectively. Their mean foot length was $25.3 \pm 1.7 \mathrm{~cm}$. None of the subjects had any known history of postural or skeletal disorders. All subjects gave informed consent according to the procedures approved by the Compliance Office of the Pennsylvania State University.

\subsection{Set-up}

The COP location was recorded and displayed with LabView (LabView 5.1, National Instruments Corporation, Dallas, TX, USA). The three force components $\left(F_{x}, F_{y}\right.$, and $F_{z}$, with $x, y$, and $z$ being the anterior-posterior, mediallateral, and vertical directions, respectively) and the three moment components $\left(M_{x}, M_{y}\right.$, and $\left.M_{z}\right)$ were acquired from a $40 \mathrm{~cm} \times 60 \mathrm{~cm}$ force platform (model $4060 \mathrm{~S}$ Bertec Inc., Worthington, OH, USA). The coordinates of the COP location were computed using $\mathrm{COP}_{x}=-M_{y} / F_{z}$ and $\mathrm{COP}_{y}=M_{x} / F_{z}$. The acquisition of COP location was performed using a personal computer (Pentium II, $450 \mathrm{MHz}$, Gateway 2000 Inc., N. Sioux City, USA) with a 12 bit A/D board (model AT-MIO64E-3, National Instruments Corporation). The sampling frequency was $50 \mathrm{~Hz}$.

\subsection{Procedure}

Given that one of the goals of this study was to compare the present results to those obtained in a previous study on gait [5], the design and procedure was based, to a large extent, on those used in that study. The experiment began with two control trials of $30 \mathrm{~s}$ each. These trials were performed in order to position the targets during the aiming task (for the use of a similar procedure, see [4]). The first trial was used to determine the preferred COP location during quiet standing. The second trial was used to establish the maximal range of COP displacement available along the anterior-posterior $(\mathrm{a}-\mathrm{p})$ axis. Subjects were able to reach 
$10.6 \pm 1.5 \mathrm{~cm}$ forward and $6.5 \pm 1.2 \mathrm{~cm}$ backward without falling. Due to these asymmetries (already reported in $[10,4]$ ), the targets were positioned $2 / 3$ forward and $1 / 3$ backward with respect to the preferred COP location during quiet standing.

After the completion of the control trials, subjects switched to the main part of the experiment: the reciprocal aiming task. In each trial, subjects were asked to displace their COP at a given frequency $(F)$ and amplitude $(A)$. Five $A$ 's $(3,4.5,6,7.5$, and $9 \mathrm{~cm})$ were crossed with five $F$ 's $(0.35,0.60,0.85,1.10$ and $1.35 \mathrm{~Hz})$. The changes in $A$ and $F$ were of rather comparable magnitude across conditions $(3 \mathrm{~cm} \times 3 \mathrm{~cm}=9 \mathrm{~cm}$ and $0.35 \mathrm{~Hz} \times 3.8 \mathrm{~Hz}=1.35 \mathrm{~Hz})$. All subjects performed one trial at each of the $F-A$ combinations (i.e., 25 trials in total). The order of the $F-A$ combinations was randomized for each subject. Online visual feedback of COP location along the $a-p$ axis was displayed as a cursor on a monitor adjusted at the subjects' head height. The prescribed $A$ was given by two targets displayed on the screen as two lines perpendicular to the a-p axis. The prescribed $F$ was given by the beep of a metronome. Subjects were instructed to keep time to the metronome by making the maximal excursion of their COP coincide with the beep. About twenty minutes before the experiment, a training session with varying $A$ 's and $F$ 's (10 trials randomly selected from the 25 possible $F-A$ combinations) was performed until the subjects felt comfortable with the task. At the beginning of each trial, subjects were given some time (about 10-15 s) in order to adjust their movement to the required amplitude and frequency. When their movement was stabilized, the experimenter started the data acquisition that lasted $30 \mathrm{~s}$.

\subsection{Data processing}

The COP signal was first low-pass filtered with a fourth order Butterworth filter (zero-phase lag) using a $4 \mathrm{~Hz}$ cutoff frequency since most of the power of the signal was below $2 \mathrm{~Hz}$ (for a review, see [26]). Then, using an automatic procedure, each trial was segmented into cycles. Each COP cycle was characterized by two parameters: its amplitude and frequency. The method used to segment each trial, and to compute amplitude and frequency is illustrated in Fig. 1. For each trial, the within-subject spatial (i.e., amplitude) and temporal (i.e., frequency) variability was calculated over all the available cycles $(10 \leq N \leq 40)$. Within-subject variability was then expressed in absolute and relative units. Standard deviations (SD) were used for the absolute units, while coefficients of variation $(\mathrm{CV}=\mathrm{SD} /$ mean, expressed in percent in the following) were used for the relative units. Thus, a total of four dependent variables were extracted from each trial: SD spatial, SD temporal, CV spatial, and CV temporal.

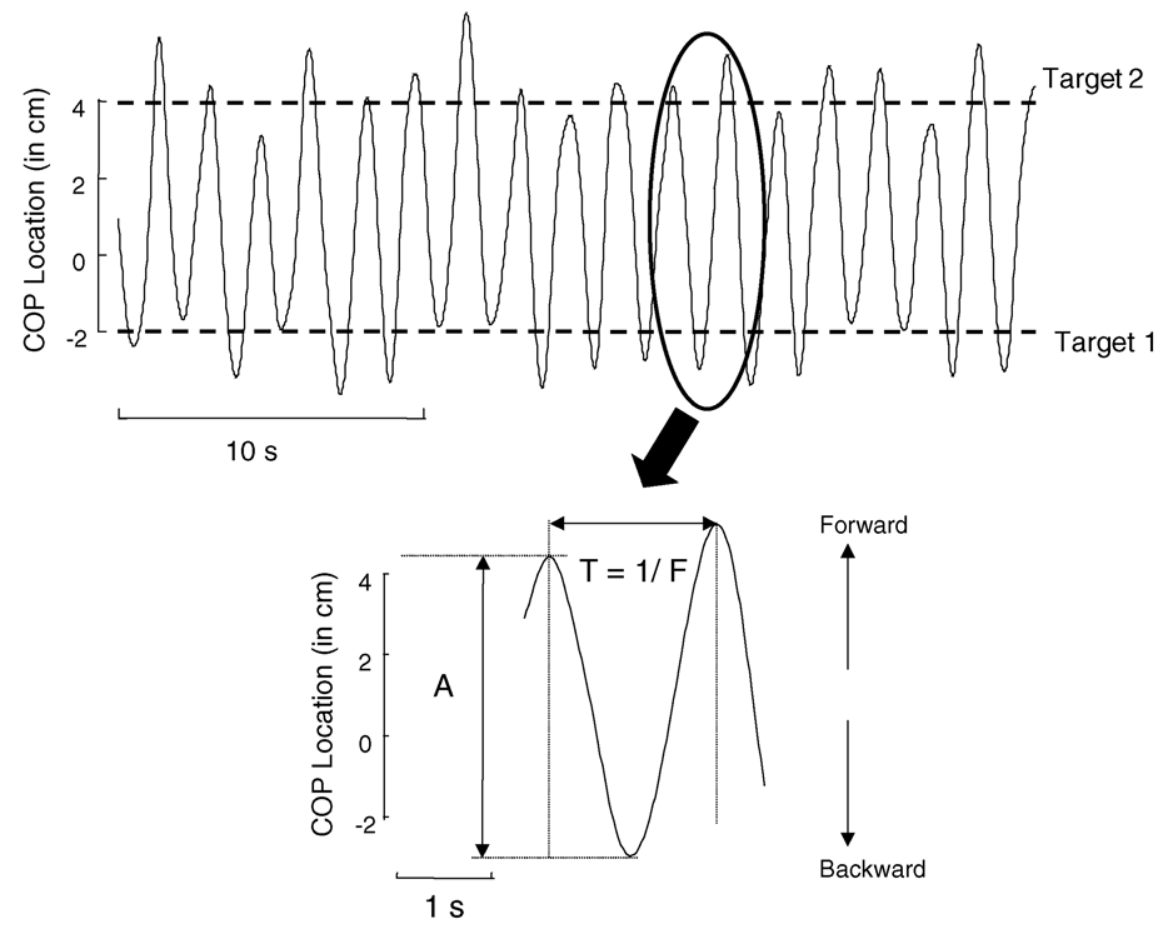

Fig. 1. Data processing of the COP location signal. The top panel presents the anterior-posterior displacement of the COP as a function of time. During this representative trial, the subject was instructed to move his COP back and forth between two targets (illustrated by the dashed lines) distant by $6 \mathrm{~cm}$, while maintaining COP oscillations at a frequency of $0.6 \mathrm{~Hz}$. The lower panel illustrates how each cycle was processed. The duration of each cycle $(T)$ was defined as the time interval between two successive peaks (i.e., extreme forward positions). The frequency of each cycle was defined as $1 / T$. The amplitude of each cycle was computed as the distance between the initial position and the extreme backward position. 

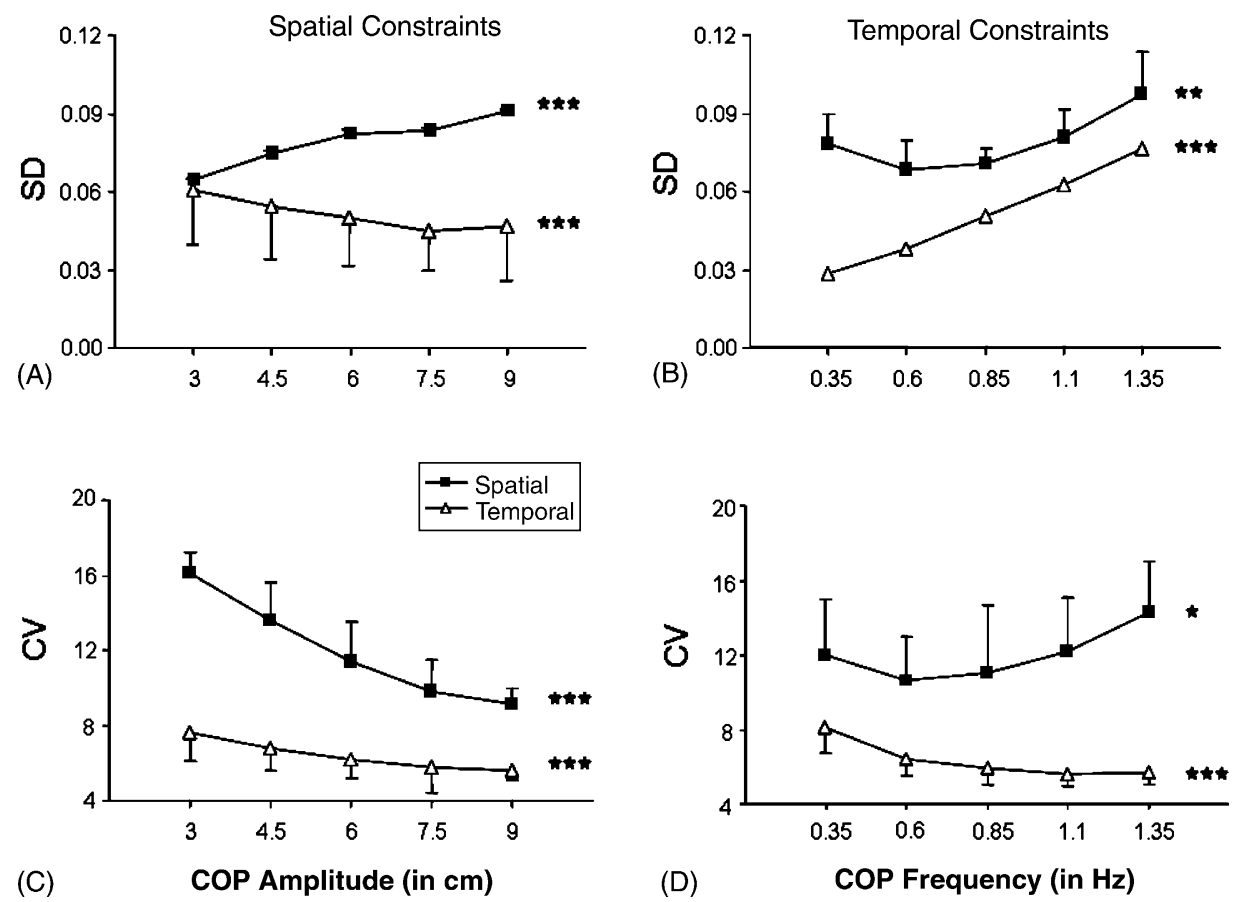

Fig. 2. Variability of COP movements as a function of COP frequency and COP amplitude. Data are averaged across experimental conditions. The effect of COP amplitude (A, C) and frequency (B, D) are presented on separate columns (left and right, respectively). Variability is either expressed by the standard deviation (SD, top row) or by the coefficient of variation (CV, bottom row). SD's are expressed in Hertz for COP frequency, and in decimeters for COP amplitude (decimeters were preferred because they allowed a single axis). The error bars represent the standard deviation across the five experimental conditions. Stars indicate when a significant main effect was observed $\left({ }^{*} p<0.05,{ }^{* * *} p<0.01,{ }^{* * * *} p<0.001\right)$.

\subsection{Statistical analysis}

Two types of statistical analysis were performed. First, the overall effects of COP amplitude (AMP) and frequency (FREQ) on spatial and temporal variability were assessed. This was achieved by employing two-way repeatedmeasures ANOVAs with AMP and FREQ as within-subject factors (5 levels each). Post-hoc Newman-Keuls analyses were used whenever necessary. Given that SD's and CVs are often not normally distributed, they were logarithmic transformed before being submitted to the ANOVAs. Second, in order to assess whether spatial and temporal variability followed the same pattern with respect to changes in COP parameters, a linear regression analysis across the 25 experimental conditions was performed. The group means were used for this analysis. For all statistical tests, the threshold of significance was set to 0.05 .

\section{Results}

Over all the 25 experimental conditions, the group mean of COP frequency never differed by more than $1 \%$ of the intended value. In contrast, the group mean COP amplitudes were consistently larger than the intended values by about $1 \mathrm{~cm}$ in all experimental conditions $(1.01 \mathrm{~cm} \pm 0.22 \mathrm{~cm})$. Collapsed across the five frequencies, effective COP amplitudes ( $\pm 1 \mathrm{SD}$ computed across frequency) were
$4.00 \pm 0.18, \quad 5.48 \pm 0.17,7.09 \pm 0.33,8.48 \pm 0.21$, and $9.99 \pm 0.24 \mathrm{~cm}$ (instead of the prescribed amplitudes of 3 , $4.5,6,7.5$ and $9 \mathrm{~cm}$, respectively).

\subsection{Spatial variability of COP movements}

Spatial variability depended largely upon the experimental conditions (see black squares in Fig. 2). The two-way ANOVA performed on absolute variability showed a main effect of AMP $[F(4,28)=11.39, p<0.001]$. This effect was related to an increase in variability with increases in amplitude (see black squares in Fig. 2A). However, when spatial variability was expressed in relative units, the effect of AMP was reversed $[F(4,28)=34.93, p<0.001]$. That is, fluctuations in COP were reduced for larger amplitudes (see black squares in Fig. 2C). As for the effect of temporal constraints, the analysis of both absolute and relative variability showed a main effect of FREQ [all $F(4,28)$ values $>3.55$, all $p$-values $<0.05$ ], reflecting a tendency to be minimal at the intermediate frequencies (see black squares in Fig. 2B and D). Post-hoc analyses revealed that absolute variability was smaller at $0.35,0.60,0.85$, and $1.10 \mathrm{~Hz}$ than at $1.35 \mathrm{~Hz}$, and that relative variability at 0.60 and $0.85 \mathrm{~Hz}$ was smaller than at $1.35 \mathrm{~Hz}$ (all $p$ values $<0.05)$. Whether variability was expressed in absolute or relative units, no significant interactions between FREQ and AMP were obtained [all $F(16,112)$ values $<0.65$, all $p$-values $>0.05]$. 


\subsection{Temporal variability of COP movements}

Temporal variability also depended largely upon the experimental conditions (see hollow triangles in Fig. 2). The two-way ANOVA conducted on absolute and relative temporal variability revealed a main effect of AMP [all $F(4,28)$ values $>7.51$, all $p$-values $<0.001]$. In both cases, fluctuations in time were smaller for larger movements (see hollow triangles in Fig. 2A and C). There were also effects of FREQ, however the nature of those effects was different for variability expressed in absolute and relative units. Indeed, when expressed in $\mathrm{Hz}$, variability increased as a function of FREQ [ $F(4,28)=49.84, p<0.001$; see hollow triangles in Fig. 2B]. In contrast, when expressed in percent, variability decreased as a function of FREQ $[F(4,28)=11.84$, $p<0.001$; see hollow triangles in Fig. 2D]. As was the case for spatial variability, there were no significant interactions between FREQ and AMP [all $F(16,112)$ values $<0.69$, all $p$-values $>0.05]$.

\subsection{Relationship between spatial and temporal variability}

Changes in temporal and spatial variability were observed across the 25 experimental conditions. It was therefore assessed whether those changes tended to increase (or decrease) in concert. Data averaged across subjects are presented in Fig. 3. Overall, very weak correlations were found between temporal and spatial variability. An analysis of absolute variability showed no significant correlation between temporal and spatial SD's $(R=0.294 ; p>0.05)$. For $\mathrm{CVs}$, the coefficient of correlation was higher, and it just reached significance $(R=0.425 ; p=0.04)$. Additional analyses performed at the subject level showed that $R$ values only reached significance for one subject (after $R$ to Fisher $z$ ' transformation, group $R=0.24 \pm 0.25$ ).

Finally, as can be seen in the lower part of Fig. 2, temporal variability was overall lower than spatial variability. The average temporal $\mathrm{CV}$ and spatial $\mathrm{CV}$ across subjects and conditions were 6.4 and $12 \%$, respectively. A paired $t$-test computed on the basis of the 25 mean temporal and spatial CVs showed that this difference was significant $(t(24)=10.31 ; p<0.001)$. $t$-Tests performed at the individual level confirmed a similar effect for each of the 8 subjects (all $t(24)$ values $>4.28$; all $p$-values $<0.001$ ).

\section{Discussion}

The goal of this study was to investigate the role of spatial and temporal constraints on COP variability during reciprocal aiming movements. Based on the present results, the following points can be made: (1) spatial and temporal constraints affect both spatial and temporal COP variability; (2) temporal and spatial COP variability are poorly correlated; and (3) relative COP temporal variability is smaller than relative COP spatial variability. In what
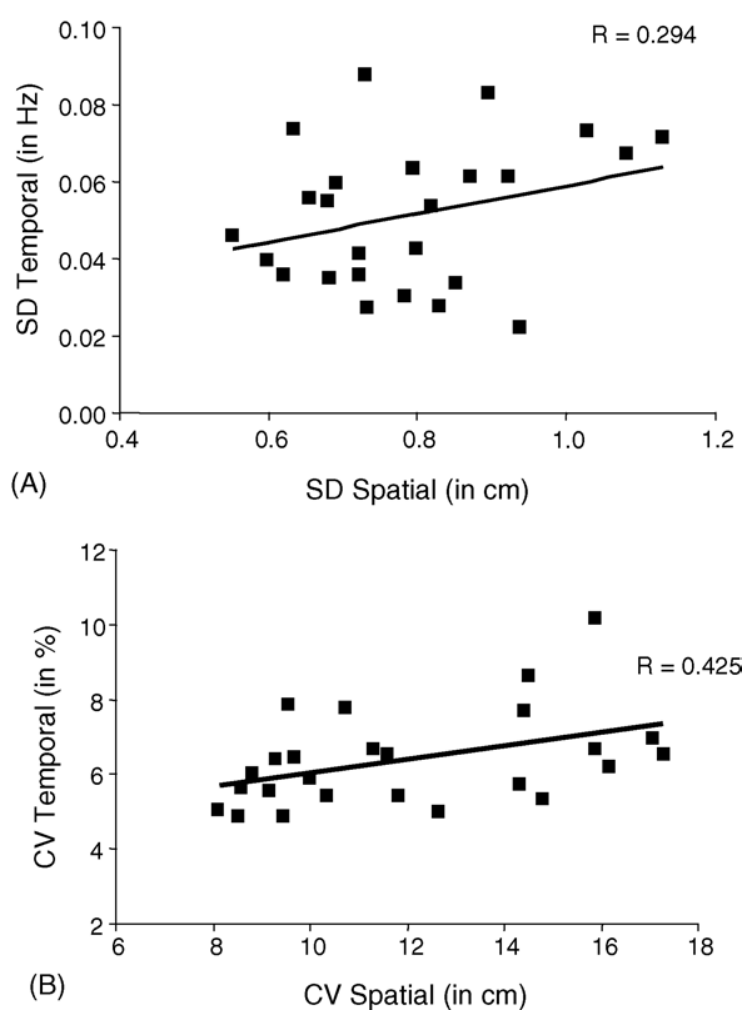

Fig. 3. Regression analysis between the temporal and spatial variability of COP movements. The regression is based on the mean of the group in each of the 25 experimental conditions. Variability is either expressed by the standard deviation $(\mathrm{SD}$, panel $\mathrm{A})$ or by the coefficient of variation $(\mathrm{CV}$, panel B).

follows, each of these issues will be discussed in more detail, in particular with regard to what is known about the control of COP location in whole-body aiming tasks [4] and stride variability in gait [5].

\subsection{The effect of spatial constraints}

The present set of data showed a clear effect of COP amplitude on COP variability. Except for absolute spatial variability (see below), all variability indices decreased as a function of movement amplitude. In other words, subjects tended to be more consistent when they performed larger COP movements. This phenomenon is in line with the study by Danion et al. [4] in which subjects performed whole-body aiming movements at different scales with their COP. However, the novelty of the present findings is to show that larger scales do not only benefit movement consistency in the space domain, but also in the time domain. What can be the reasons leading to higher consistency of the movement at large amplitudes? One possibility is that the inherent variability of the postural control system (about $1 \mathrm{~cm}$ during quiet standing) is a limiting factor of performance at small scales [4]; at large scales, the influence of this factor should be less obvious. More generally, it is remarkable that a similar conclusion (higher consistency for large movements) was reached when the effect of stride amplitude on stride 
variability was investigated [5]. The generality of this finding suggests that the effect of movement amplitude may be independent of the motor apparatus involved in the task.

At the spatial level, apparently contradictory results were observed: while absolute variability increased, relative variability decreased with movement amplitude. The fact that absolute spatial variability increased as function of movement amplitude is consistent with the study of Duarte and Zatsiorsky [8]. In that study, subjects were asked to maintain their COP immobile at various locations within the base of support. The results showed that COP steadiness, quantified by the COP area, increased when subjects occupied leaning postures. In other words, as in the present study, absolute COP variability increased as subjects moved away from the neutral COP location. To account for this phenomenon, Duarte and Zatsiorsky [8] proposed that leaning postures are associated with higher levels of muscle activation [25] which, in turn, are generally associated with higher variability in force output, and/or that humans are generally more used and adapted to the erect posture than to leaning postures. Nevertheless, subjects in the present experiment performed a dynamic task as opposed to the static task in Duarte and Zatsiorsky's study. Therefore, other factors such as inertia and difficulty in controlling the COP position at higher speeds may have also contributed to the higher variability observed at larger amplitudes. This issue aside, the fact that absolute spatial variability increased while relative spatial variability decreased is not contradictory, as it simply implies that absolute variability increases at a slower rate than COP amplitude.

\subsection{The effect of temporal constraints}

The effect of COP frequency was also demonstrated by the present study. However, this effect differed for spatial and temporal variability. At the temporal level, absolute variability was found to increase as a function of COP frequency, whereas relative variability decreased as a function of COP frequency. (A similar phenomenon was obtained with the effect of COP amplitude on spatial variability.) As before, these data imply that absolute variability increases with COP frequency, but at a slower rate than COP frequency. In any event, these contrasting patterns stress the importance of paying attention to which variability index (SD or CV) one decides to use.

In contrast with temporal variability, the effect of COP frequency on spatial variability could not be described by a monotonic function. Instead, spatial variability appeared to follow a U-shaped function of COP frequency, with maximal steadiness at $0.6 \mathrm{~Hz}$. This observation is consistent with the hypothesis formulated in the Introduction. Indeed, it had been expected that COP variability would be reduced for COP oscillations close to the resonance frequency of the postural system. Could $0.6 \mathrm{~Hz}$ correspond to the resonance frequency of the postural system in the present task? This could be the case. Indeed, it has been shown that when subjects behave like an inverted pendulum by oscillating voluntarily around the ankle, the resonance frequency of the postural system is close to $0.5 \mathrm{~Hz}$ [16]; note that the closest frequency to this value in the current design was $0.6 \mathrm{~Hz}$. This observation suggests that the subjects in this experiment may have performed the task by using such a strategy. However, given that the kinematics of the lower limbs was not recorded, further data collection will be needed to address this issue.

\subsection{Movement variability in gait and posture}

One motivation for the present study was to investigate whether earlier results obtained for stride variability [5] would generalize to other motor activities such as postural control. The following similarities between both types of motor tasks were observed: (1) temporal and spatial constraints had an effect on temporal and spatial variability; (2) movement variability was reduced under large amplitudes; (3) the effect of spatial and temporal constraints were independent; and (4) spatial variability was greater than temporal variability. Despite these numerous similarities, several major differences were also observed. First, spatial and temporal variability were poorly correlated in the postural task, whereas they were significantly correlated in the locomotion task. Second, in the postural task, the effect of temporal constraints were distinct for spatial and temporal variability (see panels B and C of Fig. 2), while they were identical in the locomotion task (both types of variability were minimal at a specific frequency). Overall, this comparison suggests that temporal and spatial constraints are both crucial parameters, however the effects of spatial constraints appear to be relatively task independent, whereas the effects of temporal constraints depend on the nature of the motor task performed.

What could be the reason(s) underlying the slightly different patterns of temporal and spatial variability in postural and locomotion tasks? One possibility could relate to differences in mechanical factors. During walking, most of the mechanical energy is provided by the kinetic energy stored in the upper body (70\% of total mass, [2]). As a result, this large amount of kinetic energy acts against fluctuations in walking speed (stride frequency $\times$ stride amplitude). That is, when stride amplitude becomes suddenly smaller, stride frequency tends to increase. In other words, large fluctuations in stride amplitude are likely to be coupled with large fluctuations in frequency (a phenomenon reported in [5]). In contrast, during a postural task, such coupling would be less encouraged because, depending on the phase of the movement, kinetic energy can be very low.

\subsection{Concluding comments}

Along with the study of Danion et al. [5], the present study demonstrates that the variability of reciprocal whole-body movements is strongly influenced by movement parameters 
such as amplitude and frequency. Concerning amplitude, larger movements are generally associated with higher consistency. Concerning frequency, there exists, in most cases, an optimal frequency leading to maximal steadiness. However, the differences in results between this and previous studies [5] indicate that certain aspects of movement variability are task dependent. This view is consistent with other studies showing task-dependent effects when comparing temporal variability of upper limb actions such as finger tapping and circle drawing $[29,23]$. Finally, it is worth stressing that clinicians and researchers interested in the issue of movement variability should be cautious when selecting their variability indices, since absolute and relative variability can lead to apparently conflicting results.

\section{References}

[1] Cattaneo D, Cardini R. Computerized system to improve voluntary control of balance in neurological patients. CyberPsychol Behav 2001;4:687-94.

[2] Cavagna GA, Thys H, Zamboni A. The sources of external work in level walking and running. J Physiol 1976;262:639-57.

[3] Collins JJ, De Luca CJ. Open-loop and closed-loop control of posture: a random walk analysis of center of pressure trajectories. Exp Brain Res 1993;95:308-18.

[4] Danion F, Duarte M, Grosjean M. Fitts' law in human standing: the effect of scaling. Neurosci Lett 1999;277:131-3.

[5] Danion F, Varraine E, Bonnard M, Pailhous J. Stride variability in human gait: the effect of stride frequency and stride length. Gait Posture 2003;18:69-77.

[6] Darling WG, Cole KJ, Abbs JH. Kinematic variability of grasp movements as a function of practice and movement speed. Exp Brain Res 1988;73:225-35.

[7] Dault MC, de Haart M, Geurts AC, Arts IM, Nienhuis B. Effects of visual center of pressure feedback on postural control in young and elderly healthy adults and in stroke patients. Human Movement Sci 2003;22:221-36.

[8] Duarte M, Zatsiorsky V. Effects of body lean and visual information on the equilibrium maintenance during stance. Exp Brain Res 2002;146:60-9.

[9] Fitts PM. The information capacity of the human motor system in controlling the amplitude of movement. J Exp Psychol (HPP) 1954;47: 381-91.

[10] Hamman RG, Mekjavic I, Mallinson AI, Longridge NS. Training effects during repeated therapy sessions of balance training using visual feedback. Arch Phys Med Rehab 1992;73:738-44.
[11] Hirvonen TP, Aalto H, Pyykkö I. Stability limits for visual feedback posturography in vestibular rehabilitation. Acta Otolaryngol 1997;529:104-7.

[12] Jacono M, Casadio M, Morasso PG, Sanguineti V. The sway-density curve and the underlying postural stabilization process. Motor Control 2004;8:292-311.

[13] Krishnamoorthy V, Latash ML, Scholz JP, Zatsiorsky VM. Muscle synergies during shifts of the center of pressure by standing persons. Exp Brain Res 2003;152:281-92.

[14] Latash ML, Ferreira SS, Wieczorek SA, Duarte M. Movement sway: changes in postural sway during voluntary shifts of the center of pressure. Exp Brain Res 2003;150:314-24.

[15] Maurer C, Peterka RJ. A new interpretation of spontaneous sway measures based on a simple model of human postural control. J Neurophysiol.

[16] McCollum G, Leen TK. Form and exploration of mechanical stability limits in erect stance. J Motor Behav 1989;21:225-44.

[17] Meyer DE, Abrams RA, Kornblum S, Wright CE, Smith JE. Optimality in human motor performance: ideal control of rapid aimed movements. Psychol Rev 1988;95:340-70.

[18] Newell KM, Carlton LG. On the relationship between peak force and peak force variability in isometric tasks. J Motor Behav 1985;17:230 41.

[19] Newell KM, Carlton LG. Force variability in isometric responses. J Exp Psychol Human Percept Perform 1988;14:37-44.

[20] Newell KM, Corcos DM. Variability and Motor Control. Champaign, IL: Human Kinetics Publishers, 1993.

[21] Nichols DS. Balance retraining after stroke using force platform biofeedback. Phys Therapy 1997;77:553-8.

[22] Schmidt RA, Zelaznik HN, Hawkins B, Frank JS, Quinn Jr JT. Motor output variability: a theory for the accuracy of rapid motor acts. Psychol Rev 1979;86:415-51.

[23] Spencer RM, Zelaznik HN. Weber (slope) analyses of timing variability in tapping and drawing tasks. J Motor Behav 2003;35:371-81.

[24] Shumway-Cook A, Anson D, Haller S. Postural sway biofeedback: its effect on reestablishing stance stability in hemiplegic patients. Arch Phys Med Rehab 1989;70:755-62.

[25] Sinha T, Maki BE. Effect of forward lean on postural ankle dynamics. IEEE Trans Rehab Eng 1996;4:348-59.

[26] Winter DAABC. Anatomy, biomechanics and control of balance during standing and walking. Waterloo, Canada: Waterloo Biomechanics, 1995.

[27] Winter DA, Patla AE, Prince F, Ishac M, Gielo-Perczak K. Stiffness control of balance in quiet standing. J Neurophysiol 1998;80:1211-21.

[28] Zatsiorsky VM, Duarte M. Instant equilibrium point and its migration in standing tasks: rambling and trembling components of the stabilogram. Motor Control 1999;3:28-38.

[29] Zelaznik HN, Spencer RM, Doffin JG. Temporal precision in tapping and circle drawing movements at preferred rates is not correlated: further evidence against timing as a general-purpose ability. J Motor Behav 2000;32:193-9. 